J. Korean Math. Soc. 50 (2013), No. 1, pp. 81-93

http://dx.doi.org/10.4134/JKMS.2013.50.1.081

\title{
A CELL BOUNDARY ELEMENT METHOD FOR A FLUX CONTROL PROBLEM
}

\author{
Youngmok Jeon And Hyung-Chun Lee
}

\begin{abstract}
We consider a distributed optimal flux control problem: finding the potential of which gradient approximates the target vector field under an elliptic constraint. Introducing the Lagrange multiplier and a change of variables the Euler-Lagrange equation turns into a coupled equation of an elliptic equation and a reaction diffusion equation. The change of variables reduces iteration steps dramatically when the GaussSeidel iteration is considered as a solution method. For the elliptic equation solver we consider the Cell Boundary Element (CBE) method, which is the finite element type flux preserving methods.
\end{abstract}

\section{Introduction}

In this article, we consider a distributed optimal control problem for a second order partial differential equation: seek $(u, p) \in H^{1}(\Omega) \times L^{2}(\Omega)$ which minimizes the cost functional

$$
\mathcal{J}(u, p)=J_{u_{d}}(u)+\delta N(p)
$$

subject to

$$
-\nabla \cdot(a \nabla u)=p \quad \text { on } \Omega,
$$

where $J_{u_{d}}(u)$ and $N(p)$ are some (semi)norms of $u$ and $p$, respectively and the parameter, $\delta$ is a positive regularization parameter. We assume that $\Omega=$ $\cup_{j=1}^{J} \Omega_{j}$ is a simply connected polygonal domain with the boundary $\Gamma$. The permeability coefficient, $a$ is a positive definite, symmetric tensor and it is constant on each subdomain $\Omega_{j}$.

In our problem the target function $u_{d} \in H^{1}(\Omega)$ will be used to provide the target flux field $\nabla u_{d}$ and the Dirichlet boundary condition $u=u_{d}$ on $\Gamma$. Throughout this article we assume $u=u_{d}=0$ on $\Gamma$ to simplify our analysis.

Received November 22, 2011; Revised April 9, 2012.

2010 Mathematics Subject Classification. 65M55, 65N30.

Key words and phrases. cell boundary element method, optimal control problem, GaussSeidel iteration.

This work was supported by Basic Science Research Program through the National Research Foundation of Korea(NRF) (grant number NRF 2010-0026032). 
Thus, we consider the gradient tracking functional

$$
J_{u_{d}}(u)=\frac{1}{2} \int_{\Omega} a \nabla\left(u-u_{d}\right) \cdot \nabla\left(u-u_{d}\right) d x
$$

The second term of the cost functional is introduced in order to bound the control function and to prove the existence of an optimal control. In general,

$$
N(p)=\frac{1}{2} \int_{\Omega} p^{2} d x
$$

The distributed optimal control problem can be derived from many physical applications $[2,4,5]$. For a subsurface flow problem, we are looking for the optimal solution $u$ and the source distribution $p$ so that the gradients of a potential flow approximate the target vector field optimally in a certain measure. In approximation of the optimality solution the flux conservation property of numerical solutions is very important. In transport problems of substances embedded in a potential flow, the distribution of substances satisfies a time dependent convection diffusion equation driven by the gradients of the flow [8]. In this case flux preserving approximation of gradients for the potential flow is crucial in obtaining physically relevant time evolution of substance distribution. For this reason, we introduce a Cell Boundary Element (CBE) numerical method for the optimal control problem. The CBE method is introduced by Jeon et al. $[6,7]$ and it can be understood as a finite element version of the finite volume method (it is different from the finite volume element method). The main advantage of the CBE method is that it is a flux preserving numerical method while it does not need a costly covolume generation.

The main contributions of the paper can be summarized as follows: firstly, a change of variables is introduced to transform the Euler-Lagrange equation to an equivalent computing friendly form. The transformed equation reduces the number of iteration dramatically when it is combined with the Gauss-Seidel iteration, especially for nonlinear problems. Secondly, the CBE method is used as a numerical method for the control problem.

The paper is organized as follows. In Section 2 we derive the optimality equations and introduce a change of variables to transform it to a computing friendly system of equations. The transformed equations are composed of a standard elliptic equation and a reaction dominated diffusion equation. In Section 3 numerical methods for elliptic equations are introduced and analyzed, the CBE method for the elliptic equation and the lowest Crouzeix-Raviart finite element method for the reaction diffusion equation. In Section 4 numerical analysis for the optimality equations are presented. In Section 5 we consider a nonlinear optimal control problem and a Gauss-Seidel iteration method is introduce. In Section 6 we present our numerical examples for linear and nonlinear optimal problems. 


\section{The optimality system}

Firstly, we introduce the standard Sobolev space, $H^{s}(\Omega)(s \geq 0)$ with the norm $\|\cdot\|_{s, \Omega}$. The $L_{2}$ space is the space of square integrable functions. The space $H_{0}^{s}(\Omega)$ is the subspace of $H^{s}(\Omega)$ with null trace. By the nature of the problem, we consider a specialized norm:

$$
H_{a}^{1}(\operatorname{div} ; \Omega)=\left\{u \in H^{1}(\Omega): \nabla \cdot(a \nabla u) \in L_{2}(\Omega)\right\} .
$$

Introducing a Lagrange multiplier, the constrained minimization problem (1.1)-(1.2) becomes a min-max problem of the functional:

$$
\begin{aligned}
L(u, p, \lambda)= & \frac{1}{2} \int_{\Omega} a \nabla\left(u-u_{d}\right) \cdot \nabla\left(u-u_{d}\right) d x+\frac{\delta}{2} \int_{\Omega} p^{2} d x \\
& +\int_{\Omega}(-\nabla \cdot(a \nabla u)-p) \lambda d x .
\end{aligned}
$$

Then, the Euler-Lagrange equation (the first optimality condition),

$$
\frac{\partial L}{\partial u}=0, \quad \frac{\partial L}{\partial p}=0 \quad \text { and } \quad \frac{\partial L}{\partial \lambda}=0,
$$

induces a coupled system of differential equations: for $(u, \lambda) \in H_{a}^{1}(\operatorname{div} ; \Omega) \cap$ $\left.H_{0}^{1}(\Omega)\right) \times L_{2}(\Omega)$,

$(2.2)\left(a \nabla\left(u-u_{d}\right), \nabla v\right)_{\Omega}+(-\nabla \cdot(a \nabla v), \lambda)_{\Omega}=0, \quad v \in H_{a}^{1}(\operatorname{div} ; \Omega) \cap H_{0}^{1}(\Omega)$,

$$
\begin{array}{cl}
\delta(p, h)_{\Omega}-(h, \lambda)_{\Omega}=0, & h \in L_{2}(\Omega), \\
(-\nabla \cdot(a \nabla u)-p, \mu)_{\Omega}=0, & \mu \in L_{2}(\Omega),
\end{array}
$$

where $(u, v)=\int_{\Omega} u v d \Omega$.

To derive a strong form of differential equations, we impose a stronger regularity and an artificial boundary condition for the Lagrange multiplier, that is, $\lambda \in H_{a}^{1}(d i v ; \Omega) \cap H_{0}^{1}(\Omega)$. From (2.3) we set

$$
p=\frac{\lambda}{\delta} \text {. }
$$

The integration by parts with the boundary conditions $v=\lambda=0$ on $\Gamma$ yields

$$
\begin{aligned}
-\nabla \cdot(a \nabla u)-\nabla \cdot(a \nabla \lambda) & =-\nabla \cdot\left(a \nabla u_{d}\right), \\
-\nabla \cdot(a \nabla u)-\frac{\lambda}{\delta} & =0,
\end{aligned}
$$

on $\Omega$ with the boundary condition $u=\lambda=0$ on $\Gamma$.

Note that the Gataux differentiability for the Lagrangian can easily be proven (see $[2,4]$ and references therein).

Introducing a new variable $w$ so that $w=u+\lambda$, we can have the following computation friendly form of the equations (2.6) and (2.7): find $(w, \lambda, u) \in$ $H_{a}^{1}(\operatorname{div} ; \Omega) \times\left(H_{a}^{1}(\operatorname{div} ; \Omega) \times H_{0}^{1}(\Omega)\right) \times H_{a}^{1}(\operatorname{div} ; \Omega)$ that satisfies

$$
\begin{aligned}
-\nabla \cdot(a \nabla w) & =f_{d}, \\
\delta \nabla \cdot(a \nabla w)+\{-\delta \nabla \cdot(a \nabla \lambda)+\lambda\} & =0,
\end{aligned}
$$




$$
-w+\lambda+u=0
$$

on $\Omega$ and the boundary conditions $w=\lambda=0$ on $\Gamma$, where $f_{d}=-\nabla \cdot\left(a \nabla u_{d}\right)$. Introduction of a new variable $w$ is very important when one tries to solve a nonlinear optimal control problem (see Section 5). Applying the GaussSeidel iteration in the equations (2.6) and (2.7) may be costly, while the GaussSeidel iteration for the equations (2.8)-(2.10) provides a stable, fast convergent numerical scheme.

We suggest the Gauss-Seidel iteration for the equation (2.8)-(2.10) as follows:

$$
\begin{aligned}
-\nabla \cdot\left(a \nabla w^{n+1}\right) & =f_{d}, \\
-\delta \nabla \cdot\left(a \nabla \lambda^{n+1}\right)+\lambda^{n+1} & =\delta\left\{-\nabla \cdot\left(a \nabla w^{n+1}\right)\right\}, \\
u^{n+1} & =w^{n+1}-\lambda^{n+1},
\end{aligned}
$$

with the boundary conditions, $w^{n+1}=0$ and $\lambda^{n+1}=0$ on $\Gamma$.

Indeed, the above Gauss-Seidel iteration ends at one iteration for a linear problem.

\section{Numerical methods for elliptic equations}

In this paper we are looking for the almost flux conserving numerical solution for the optimal problem under the assumption that the regularization parameter $\delta$ is small. For that we consider the Cell Boundary Element (CBE) method for (2.11) and the nonconforming finite element method (NcFEM) for (2.12). Since the NcFEM is not a flux preserving method, our method will not be exactly flux preserving. However, in view of the equation (2.12), the exact solution $\lambda$ will satisfy the estimate, $\sqrt{\delta}|\lambda|_{1, \Omega}+\|\lambda\|_{0, \Omega} \leq C \delta$. Therefore, using the NcFEM for the equation (2.12) will have a very little influence on flux conservation property of the other solutions $u$ and $w$ when the parameter $\delta$ is a small positive number.

In this section we review the CBE method and the NcFEM, briefly and detailed description on those method will be found $[6,7]$ and [1], respectively.

Let $\mathcal{T}_{h}$ be the regular triangulation of $\Omega=\cup_{j=1}^{J} \Omega_{j}$ and $\Gamma_{h}$ the discretization of $\Gamma$, induced by $\mathcal{T}_{h}$. Then $\mathcal{E}_{h}$ and $\mathcal{E}_{h}^{0}$ denote the set of edges and interior edges generated by $\mathcal{T}_{h}$, respectively, and $\mathcal{M}_{h}$ denotes the set of midpoints of edges. The skeleton of a triangulation $\mathcal{T}_{h}$ is $\mathcal{K}_{h}=\cup_{e \in \mathcal{E}_{h}} e$.

Introduce the nonconforming $P_{1}$ approximation space for $v$ :

$$
S_{h}=\left\{v \in \oplus_{T \in \mathcal{T}_{h}} S_{T}: v \text { is continuous on } \mathcal{M}_{h} \text { and } v(p)=0 \text { for } p \in \Gamma_{h} \cap \mathcal{M}_{h}\right\}
$$

with $S_{T}=\operatorname{span}\{1, x, y\}$. Then, there is a natural interpolation $I_{h}: C(\Omega) \rightarrow S_{h}$ such that

$$
I_{h}(u)(x)=\sum_{m \in \mathcal{M}_{h}} u(m) \phi_{m}(x)
$$


We also introduce discrete Sobolev seminorms and norms; for $k \in \mathbb{Z}$,

$$
|u|_{k, h}^{2}=\sum_{T \in \mathcal{T}_{h}}|u|_{k, T}^{2}, \quad\|u\|_{k, h}^{2}=\|u\|_{0, \Omega}^{2}+\sum_{T \in \mathcal{T}_{h}}|u|_{k, T}^{2} .
$$

The analysis for the reaction diffusion equation is based on the following norm:

$$
\|u\|_{\delta, h}^{2}=\delta|u|_{1, h}^{2}+\|u\|_{0, \Omega}^{2} .
$$

\subsection{The cell boundary element method for the elliptic equation}

Consider a Dirichlet problem: find $u \in H_{0}^{1}(\Omega)$ such that

$$
-\nabla \cdot(a \nabla u)=f \text { in } \Omega,
$$

where $f$ is a piecewise constant function, $f \in L_{2}(\Omega)$. When $f$ is not a piecewise constant function we approximate $f$ by the volume preserving approximation, that is, $\left.f_{h}\right|_{T}=\frac{1}{|T|} \int_{T} f d x$. If $f \in L_{2}(\Omega)$, this approximation introduces error of the order $O(h)$ in the energy norm. Therefore, the overall approximation property of $u$ does not change with the $P_{1}$ CBE method.

To derive the CBE method we consider the localized problem of (3.5):

$$
\begin{aligned}
-\nabla \cdot(a \nabla u) & =f \quad \text { in } T, \\
{[[\nabla u]] } & =0 \quad \text { on } e \in \mathcal{E}_{h}^{0} .
\end{aligned}
$$

The jump of normal fluxes is defined as follows:

$$
[[\nabla u]] \equiv\left\{\begin{array}{cc}
(a \nabla u) \cdot \nu+\left(a^{\prime} \nabla u\right) \cdot \nu^{\prime}, & e=\partial T \cap \partial T^{\prime}, \\
(a \nabla u) \cdot \nu, & e=\partial T \cap \Gamma_{h},
\end{array}\right.
$$

where $\nu$ and $\nu^{\prime}$ are the unit outward normal vectors on $\partial T$ and $\partial T^{\prime}$, respectively. The solution $u$ of (3.6) allows locally the following superposition:

$$
u=v+G\left(f_{h}\right) \quad \text { on } T \text {. }
$$

Here $v$ satisfies $-\nabla \cdot(a \nabla v)=0$ on $T$ and $v=u$ on $\partial T$, and $G(f)$ is a Green bubble function such that $-\nabla(a \nabla(G(f))=f$ on $T$ and $G(f)=0$ on $\partial T$. Then we have

$$
[[\nabla u]]=[[\nabla v]]+[[\nabla G(f)]] \quad \text { on } e \in \mathcal{E}_{h} .
$$

Using flux continuity on cell interfaces, we have

$$
\int_{e}[[\nabla v]] d s=-\int_{e}[[\nabla G(f)]] d s, \quad e \in \mathcal{E}_{h}^{0} .
$$

Introducing a piecewise constant test function on $\mathcal{K}_{h}$ such that $\overline{w_{h}}=\frac{1}{|e|} \int_{e} w_{h} d s$ with $w_{h} \in S_{h}$, we can rewrite the above equation:

$$
\int_{\mathcal{K}_{h}}[[\nabla v]] \overline{w_{h}} d s=-\int_{\mathcal{K}_{h}}[[\nabla G(f)]] \overline{w_{h}} d s, \quad w_{h} \in S_{h} .
$$

Let us introduce the natural interpolation $I_{h, T}: C(\bar{T}) \rightarrow S_{T}$. As long as there is no risk of misunderstanding, we denote $I_{h, T}$ by $I_{h}$ for notational 
simplicity. Then we consider approximation of the bubble function $G(f)$. Let $a=\left[\begin{array}{ll}a_{11} & a_{12} \\ a_{21} & a_{22}\end{array}\right]$ on $T$. Then we may take

$$
G_{h}(f)=F(f)-I_{h}(F(f)) \quad \text { with } F(f)=\frac{f}{2\left(a_{11}+a_{22}\right)}\left(x^{2}+y^{2}\right)
$$

on $T$. Then we have $G_{h}(f) \approx G(f)$ and $-\nabla \cdot\left(a \nabla G_{h}(f)\right)=f$ on $T$. Note that $f$ is constant on each $T$.

The $P_{1}$ CBE method is to find $v_{h} \in S_{h}$ such that

$$
\int_{\mathcal{K}_{h}}\left[\left[\nabla v_{h}\right]\right] \overline{w_{h}} d s=-\int_{\mathcal{K}_{h}}\left[\left[\nabla G_{h}(f)\right]\right] \overline{w_{h}} d s .
$$

After $v_{h}$ being obtained, we obtain the approximate solution and its flux as follows.

$$
u_{h}=v_{h}+G_{h}(f), \quad a \nabla u_{h}=a \nabla v_{h}+a \nabla G_{h}(f) .
$$

Now we introduce some results on numerical analysis [6]. Theorem 3.1 asserts that the CBE has the same stiffness matrix as the Crouzeix-Raviart $P_{1}$ finite element method, while the right hand side is different by $D_{h}$. Theorem 3.2 provides a convergence result.

Theorem 3.1. The stiffness matrix and the right hand side of the equation (3.11) satisfy the following relations: for $v_{h}, w_{h} \in S_{h}$

$$
\int_{\mathcal{K}_{h}}\left[\left[\nabla v_{h}\right]\right] \bar{w}_{h} d s=\left(a \nabla v_{h}, \nabla w_{h}\right)_{h}
$$

and

$$
-\int_{\mathcal{K}_{h}}\left[\left[\nabla G_{h}(f)\right]\right] \overline{w_{h}} d s=\left(f, w_{h}\right)_{h}+D_{h}\left(f, w_{h}\right)
$$

where

$$
D_{h}\left(f, w_{h}\right)=-\sum_{T \in \mathcal{T}_{h}}\left[\left(\partial_{\nu}^{a} G(f), \overline{w_{h}}-w_{h}\right)_{\partial T}+\left(a \nabla G_{h}(f), \nabla w_{h}\right)_{T}\right] .
$$

Here, $(\nabla u, \nabla v)_{h}=\sum_{T \in \tau_{h}}(\nabla u, \nabla v)_{T}$ and $\partial_{\nu}^{a} G(f)=(a \nabla G(f)) \cdot \nu$.

Proof. Since $\nabla v_{h}$ is constant on each $T$, using the integration by parts,

$$
\begin{aligned}
\int_{\mathcal{K}_{h}}\left[\left[\nabla v_{h}\right]\right] \bar{w}_{h} d s & =\int_{\mathcal{K}_{h}}\left[\left[\nabla v_{h}\right]\right] w_{h} d s \\
& =\sum_{T \in \mathcal{T}_{h}} \int_{\partial T}\left(a \nabla v_{h}\right) \cdot \nu w_{h} d s \\
& =\left(a \nabla v_{h}, \nabla w_{h}\right)_{h} .
\end{aligned}
$$

Now,

$$
\begin{aligned}
& -\int_{\mathcal{K}_{h}}\left[\left[\nabla G_{h}(f)\right]\right] \overline{w_{h}} d s \\
= & -\int_{\mathcal{K}_{h}}\left[\left[\nabla G_{h}(f)\right]\right] w_{h} d s-\int_{\mathcal{K}_{h}}\left[\left[\nabla G_{h}(f)\right]\right]\left(\bar{w}_{h}-w_{h}\right) d s
\end{aligned}
$$




$$
\begin{aligned}
& =-\sum_{T \in \mathcal{T}_{h}}\left[\left(\partial_{\nu}^{a} G(f), w_{h}\right)_{\partial T}+\left(\partial_{\nu}^{a} G(f), \bar{w}_{h}-w_{h}\right)_{\partial T}\right] \\
& =\left(f, w_{h}\right)_{\Omega}-\sum_{T \in \mathcal{T}_{h}}\left[\left(a \nabla G_{h}(f), \nabla w_{h}\right)_{T}+\left(\partial_{\nu}^{a} G(f), \bar{w}_{h}-w_{h}\right)_{\partial T}\right] .
\end{aligned}
$$

Then, the theorem is proved.

Theorem 3.2. For $f \in L_{2}(\Omega)$, let $u$ be the exact solution of (3.5) and $u_{h}$ be the $P_{1} C B E$ approximate solution. Then

$$
\left\|u-u_{h}\right\|_{1, h} \leq C h\|f\|_{0, \Omega} .
$$

Proof. The proof is based on the proof of the lowest order Crouzeix-Raviart finite element method. The lowest order Crouzeix-Raviart finite element solution, $u_{h}^{C R}$ satisfies

$$
\left(a \nabla u_{h}^{C R}, \nabla w_{h}\right)_{h}=\left(f, w_{h}\right)_{\Omega}, \quad w_{h} \in S_{h} .
$$

Simple calculation yields that

$$
\left(a \nabla\left(u_{h}-u_{h}^{C R}\right), \nabla w_{h}\right)_{h}=D_{h}\left(f, w_{h}\right) .
$$

Since $G_{h}(f)=F(f)-I_{h}(F(f))$,

$$
\left|\left(\partial_{\nu}^{a} G(f), \nabla w_{h}\right)_{T}\right| \leq C h|F(f)|_{2, T}\left|w_{h}\right|_{1, T} \leq C\|f\|_{0, T}\left|w_{h}\right|_{1, T} .
$$

Using Corollary (10.17) with the scaling argument in [1],

$$
\left|\left(a \nabla G_{h}(f), \bar{w}_{h}-w_{h}\right)_{\partial T}\right| \leq C h\left|G_{h}(f)\right|_{2, T}\left|w_{h}\right|_{1, T} \leq C h\|f\|_{0, T}\left|w_{h}\right|_{1, T} .
$$

Then

$$
\left|D_{h}\right| \leq C h\|f\|_{0, \Omega}\left\|w_{h}\right\|_{1, h}
$$

Therefore,

$$
\mid\left(\left.\nabla\left(u_{h}^{C R}-u_{h}, \nabla w_{h}\right)_{T}\left|\leq C h\|f\|_{0, T}\right| w_{h}\right|_{1, T} .\right.
$$

It is well-known that $\left\|u-u_{h}^{C R}\right\|_{1, h} \leq C h\|f\|_{0, \Omega}$. The triangle inequality with the Poincaré-Friedrichs inequality $[1,3]$ yields the desired convergence estimate.

\subsection{The nonconforming finite element method for the reaction dif- fusion equation}

The finite element method of the reaction diffusion equation is described briefly. The nonconforming $P_{1}$ finite element method for the reaction diffusion equation has advantage in that it has a mass-lumping property, which prevents non-physical oscillations in numerical solutions on the region of boundary layer [9].

Consider a reaction diffusion equation with the Dirichlet boundary condition:

$$
\begin{array}{rlll}
-\delta \nabla \cdot(a \nabla \lambda)+\lambda & =f & \text { in } \Omega, \\
\lambda & =0 & \text { on } \Gamma .
\end{array}
$$


The lowest order Crouzeix-Raviart finite element method is to find $\lambda_{h} \in S_{h}$ such that

$$
\delta\left(a \nabla \lambda_{h}, \nabla \gamma_{h}\right)_{h}+\left(\lambda_{h}, \gamma_{h}\right)_{\Omega}=\left(f, \gamma_{h}\right)_{\Omega}, \quad \gamma_{h} \in S_{h} .
$$

Then the following convergence analysis is straightforward and it is found in standard textbooks [1], [9].

Theorem 3.3. The exact and its approximate solutions, $\lambda$ and $\lambda_{h}$ satisfy the convergence estimate:

$$
\left\|\lambda-\lambda_{h}\right\|_{\delta, h} \leq C\left(h^{2}+\sqrt{\delta} h\right)\left(\|f\|_{0, \Omega}+\|\lambda\|_{0, \Omega}\right) .
$$

Proof. By the integration by parts,

$$
\delta\left(a \nabla \lambda_{h}, \nabla \gamma\right)_{h}+\left(\lambda_{h}, \gamma\right)_{\Omega}=\delta(a \nabla \lambda, \nabla \gamma)_{h}+(\lambda, \gamma)_{\Omega}+E_{h}, \quad \gamma \in S_{h},
$$

where

$$
E_{h}=-\delta \sum_{T \in \mathcal{T}_{h}}\left\langle\partial_{\nu}^{a} \lambda, \gamma\right\rangle_{\partial T}=-\delta \sum_{T \in \mathcal{T}_{h}}\left\langle\partial_{\nu}^{a} \lambda, \gamma-\bar{\gamma}\right\rangle_{\partial T}
$$

since $\partial_{\nu}^{a} \lambda$ is continuous and $\bar{\gamma}$ is single valued on $\mathcal{K}_{h}$. Using Corollary (10.17) with the scaling argument in [1], we have

$$
\begin{aligned}
\left|E_{h}\right| & \leq \delta \sum_{T \in \mathcal{T}_{h}}\left|\left\langle\partial_{\nu}^{a} \lambda, \gamma-\bar{\gamma}\right\rangle_{\partial T}\right| \\
& \leq C h \delta \sum_{T \in \mathcal{T}_{h}}|\lambda|_{2, T}|\gamma|_{1, T} \\
& \leq C h \delta|\lambda|_{2, h}|\gamma|_{1, h} .
\end{aligned}
$$

Then, for $\mu \in S_{h}$,

$$
\begin{aligned}
& \delta\left(a \nabla\left(\lambda_{h}-\mu\right), \nabla \gamma\right)_{h}+\left(\left(\lambda_{h}-\mu\right), \gamma\right)_{\Omega} \\
= & \delta(a \nabla(\lambda-\mu), \nabla \gamma)_{h}+((\lambda-\mu), \gamma)_{\Omega}+E_{h}, \quad \gamma \in S_{h} .
\end{aligned}
$$

Taking $\gamma=\lambda_{h}-\mu$, we have

$$
\begin{aligned}
\left\|\lambda_{h}-\mu\right\|_{\delta, h} & \leq \sqrt{\delta}\|\lambda-\mu\|_{1, h}+\|\lambda-\mu\|_{0, \Omega}+C \sqrt{\delta} h|\lambda|_{2, h} \\
& \leq C\left(\sqrt{\delta} h+h^{2}\right)\|\lambda\|_{2, h}
\end{aligned}
$$

for an optimal $\mu \in S_{h}$. Since $\|\lambda\|_{2, \Omega} \leq C\left(\|f\|_{0, \Omega}+\|\lambda\|_{0, \Omega}\right)[3]$, the triangle inequality yields

$$
\left\|\lambda-\lambda_{h}\right\|_{\delta, h} \leq C\left(h^{2}+\sqrt{\delta} h\right)\left(\|f\|_{0, \Omega}+\|u\|_{0, \Omega}\right) .
$$

\section{Numerical analysis of the optimal control equations}

As mentioned before, the equation (2.11) is solved by the CBE method in Subsection 3.1 and the equation (2.12) is solved by the lowest order CrouzeixRaviart finite element method in Subsection 3.2.

Finally, we prove the convergence of $\left(u_{h}, p_{h}\right)$ to the exact solution $(u, p)$. It is easy to see that the above iteration ends with one iteration. 
Theorem 4.1. Let $\left(u_{h}, p_{h}\right)$ be the approximate solution by the above GaussSeidel iteration, while $(u, p)$ is the exact solution of (2.8)-(2.10). Then we have the following convergence estimates:

$$
\begin{aligned}
\left\|u-u_{h}\right\|_{1, h} & \leq C\left(h\left\|f_{d}\right\|_{0, \Omega}+\left(\delta h+\sqrt{\delta} h^{2}\right)\|p\|_{0, \Omega}\right), \\
\left\|p-p_{h}\right\|_{0, \Omega} & \leq C\left(h^{2}+\sqrt{\delta} h\right)\left(\left\|f_{d}\right\|_{0, \Omega}+\|p\|_{0, \Omega}\right),
\end{aligned}
$$

where $f_{d}=-\nabla \cdot\left(a \nabla u_{d}\right)$.

Proof. From (2.11)-(2.13), $w$ and $p=\frac{\lambda}{\delta}$ satisfy

$$
\begin{aligned}
-\nabla(a \nabla w) & =f_{d}, \\
-\delta \nabla(a \nabla p)+p & =f_{d}
\end{aligned}
$$

on $\Omega$ with boundary conditions, $w=0$ and $p=0$ on $\Gamma$. Analysis in Section 3 assert that $w_{h}$ and $p_{h}$ have the following convergence properties:

$$
\begin{aligned}
\left\|w-w_{h}\right\|_{1, h} & \leq C h\left\|f_{d}\right\|_{0, \Omega} \\
\left\|p-p_{h}\right\|_{\delta, h} & \leq C\left(h^{2}+\sqrt{\delta} h\right)\left(\left\|f_{d}\right\|_{0, \Omega}+\|p\|_{0, \Omega}\right) .
\end{aligned}
$$

Therefore,

Since $\lambda=p \delta$,

$$
\begin{aligned}
\left\|p-p_{h}\right\|_{0, h} & \leq C\left(h^{2}+\sqrt{\delta} h\right)\left(\left\|f_{d}\right\|_{0, \Omega}+\|p\|_{0, \Omega}\right), \\
\left|p-p_{h}\right|_{1, h} & \leq C\left(h+\frac{h^{2}}{\sqrt{\delta}}\right)\left(\left\|f_{d}\right\|_{0, \Omega}+\|p\|_{0, \Omega}\right) .
\end{aligned}
$$

$$
\left|\lambda-\lambda_{h}\right|_{1, h} \leq C\left(\delta h+\sqrt{\delta} h^{2}\right)\left(\left\|f_{d}\right\|_{0, \Omega}+\|p\|_{0, \Omega}\right) .
$$

Therefore,

$$
\begin{aligned}
\left\|u-u_{h}\right\|_{1, h} & \leq\left\|w-w_{h}\right\|_{1, h}+\left\|\lambda-\lambda_{h}\right\|_{1, h} \\
& \leq C h\left\|f_{d}\right\|_{0, \Omega}+C\left(\delta h+\sqrt{\delta} h^{2}\right)\left(\left\|f_{d}\right\|_{0, \Omega}+\|p\|_{0, \Omega}\right) \\
& \leq C\left(h\left\|f_{d}\right\|_{0, \Omega}+\left(\delta h+\sqrt{\delta} h^{2}\right)\|p\|_{0, \Omega}\right) .
\end{aligned}
$$

\section{Nonlinear problem}

Let us consider a nonlinear problem: minimize the cost functional

$$
\mathcal{J}(u, p)=J_{u_{d}}(u)+\delta N(p)
$$

subject to

$$
-\nabla \cdot(a \nabla u)+\mathcal{F}(u)=p \quad \text { on } \Omega,
$$

where $\mathcal{F}$ is a nonlinear operator. By following the same process in Section 2, we obtain the strong form of optimality system: find $(u, \lambda) \in\left[H_{a}^{1}(\operatorname{div} ; \Omega) \cap H_{0}^{1}(\Omega)\right]^{2}$ such that

$$
\begin{aligned}
-\nabla \cdot(a \nabla u)-\nabla \cdot(a \nabla \lambda)+\mathcal{F}^{\prime}(u)^{*} \lambda & =f_{d}, \\
-\nabla \cdot(a \nabla u)+\mathcal{F}(u)-\frac{\lambda}{\delta} & =0 .
\end{aligned}
$$


Here, $\mathcal{F}^{\prime}(u)^{*}$ is the adjoint of $\mathcal{F}^{\prime}(u)$ and $\mathcal{F}^{\prime}$ is the Frechet derivative of $\mathcal{F}$. Introducing a new variable $w=u+\lambda$, we obtain the computing friendly form of equations:

$$
\begin{aligned}
-\nabla \cdot(a \nabla w)+\mathcal{F}^{\prime}(u)^{*} \lambda & =f_{d} \\
\delta \nabla \cdot(a \nabla w)+\{-\delta \nabla \cdot(a \nabla \lambda)+\lambda\}-\delta \mathcal{F}(u) & =0 \\
-w+\lambda+u & =0 .
\end{aligned}
$$

Then the Gauss-Seidel iteration can provide an efficient numerical algorithm for the problem as follows:

$$
\begin{aligned}
-\nabla \cdot\left(a \nabla w^{n+1}\right) & =-\mathcal{F}^{\prime}\left(u^{n}\right)^{*} \lambda^{n}+f_{d}, \\
-\delta \nabla \cdot\left(a \nabla \lambda^{n+1}\right)+\lambda^{n+1} & =\delta\left\{-\nabla \cdot\left(a \nabla w^{n+1}\right)+\mathcal{F}\left(u^{n}\right)\right\}, \\
u^{n+1} & =w^{n+1}-\lambda^{n+1} .
\end{aligned}
$$

Remark 5.1. Let us apply the Gauss-Seidel iteration directly to the system (5.3) and (5.4) as follows:

$$
\begin{aligned}
-\nabla \cdot\left(a \nabla u^{n+1}\right)-\nabla \cdot\left(a \nabla \lambda^{n}\right)+\mathcal{F}^{\prime}\left(u^{n}\right)^{*} \lambda^{n} & =f_{d}, \\
-\nabla \cdot\left(a \nabla u^{n+1}\right)+\mathcal{F}\left(u^{n+1}\right)-\frac{\lambda^{n+1}}{\delta} & =0 .
\end{aligned}
$$

\begin{tabular}{|c|c|c|c|c|c|c|c|}
\hline$n$ & $u-u_{h} \|_{0}$ & $\alpha$ & $\left|u-u_{h}\right|_{1}$ & $\alpha$ & $p-p_{h} \|_{0}$ & $\alpha$ & $\operatorname{err}\left(\right.$ flu $\left._{D}\right)$ \\
\hline 4 & $9.7728 \mathrm{e}-02$ & & $3.3098 \mathrm{e}-01$ & & $7.0505 \mathrm{e}-01$ & & $7.4093 \mathrm{e}-03$ \\
\hline 8 & $2.5181 \mathrm{e}-02$ & 1.96 & $1.5689 \mathrm{e}-01$ & 1.08 & $1.8643 \mathrm{e}-01$ & 1.92 & $7.6618 \mathrm{e}-03$ \\
\hline 16 & $6.3434 \mathrm{e}-03$ & 1.99 & $7.7309 \mathrm{e}-02$ & 1.02 & $4.7258 \mathrm{e}-02$ & 1.98 & $7.7162 \mathrm{e}-03$ \\
\hline 32 & $1.5890 \mathrm{e}-03$ & 2.00 & $3.8532 \mathrm{e}-02$ & 1.00 & $1.1852 \mathrm{e}-02$ & 2.00 & $7.7660 \mathrm{e}-03$ \\
\hline
\end{tabular}

For simplification of our discussion, we assume $\mathcal{F}=0$. Simple calculation yields

\begin{tabular}{|c|c|c|c|c|c|c|c|}
\hline$n$ & $\left\|u-u_{h}\right\|_{0}$ & $\alpha$ & $\left|u-u_{h}\right|_{1}$ & $\alpha$ & $\mid p-p_{h} \|_{0}$ & $\alpha$ & $\operatorname{err}\left(\right.$ flu $\left._{D}\right)$ \\
\hline 4 & $9.7973 \mathrm{e}-02$ & & $.3211 \mathrm{e}-01$ & & $.0603 \mathrm{e}-01$ & & $7.4971 \mathrm{e}-05$ \\
\hline 8 & $2.5244 \mathrm{e}-02$ & 1.96 & $2 \mathrm{e}-01$ & 1. & $\overline{\mathrm{e}-01}$ & 1.5 & $7.7931 \mathrm{e}-05$ \\
\hline 16 & -03 & 1.99 & 7.7 & 1.02 & 4.7 & 1.98 & 7.867 \\
\hline 22 & $1.5927 \mathrm{e}-03$ & 2.00 & $3.8603 \mathrm{e}-02$ & 1.01 & $1.1876 \mathrm{e}-02$ & 2.00 & 7.8 \\
\hline
\end{tabular}
that the sequence, $\left\{\lambda^{n}\right\}_{n=0}^{\infty}$ satisfies

$$
\lambda^{n+1}=\delta \nabla\left(a \nabla \lambda^{n}\right)+\delta f_{d}
$$

Since the eigenvalues of the operator $-\delta \nabla(a \nabla \cdot)$ is unbounded, convergence may not happen unless the initial condition $\lambda^{0}$ is properly chosen.

Table 1: Numerical results with Example 6.1: $\delta=10^{-4}$

Table 2: Numerical results with Example 6.1: $\delta=10^{-6}$

\section{Numerical examples}

The Gauss-Seidel iteration for the linear problem (1.1) and (1.2) involves solving only two elliptic equations once. For the nonlinear problem (5.1) and 

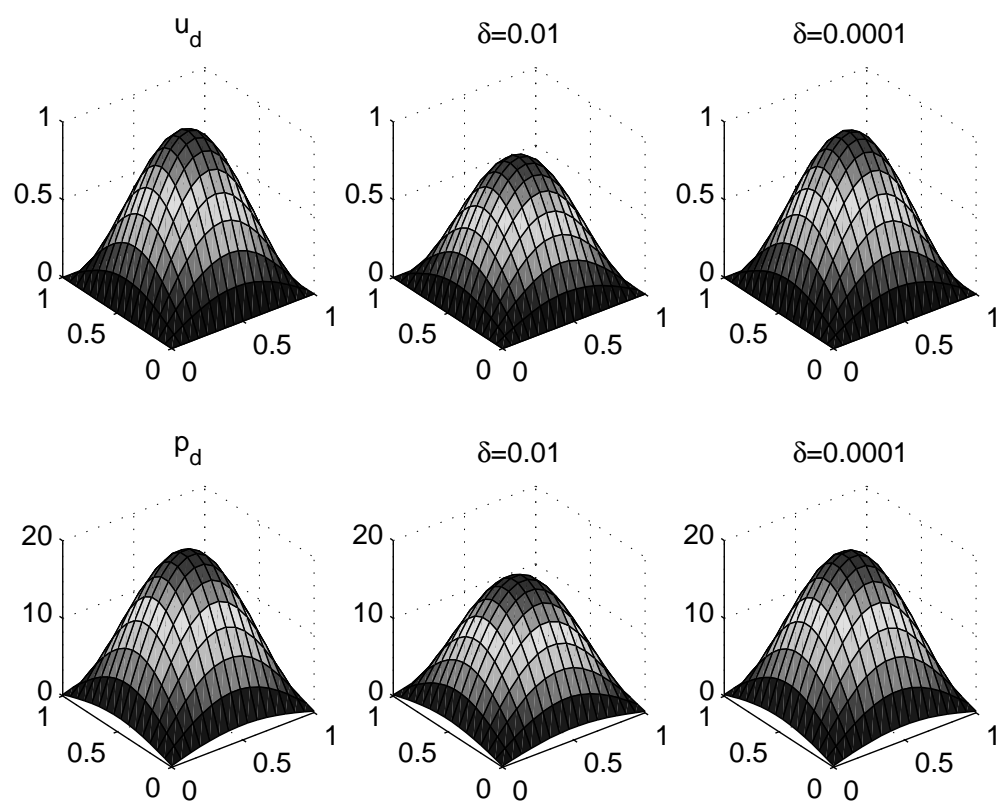

Figure 1: The controlled solutions $u_{h}$ with the target solution $u_{d}$ (top) and $p_{h}$ with the target solution $p_{d}$ (bottom).

(5.2) the Gauss-Seidel iteration requires for elliptic equations to be solved repeatedly and the algorithm (5.5)-(5.7) seems to provide a stable, fast converging numerical scheme when $\delta$ is small, at least for numerical examples considered in this section. Here, we provide both the linear and nonlinear examples.

Example 6.1. Let us consider a linear distributed optimal control problem:

$$
\min _{u \in H_{0}^{1}(\Omega)} \frac{1}{2} \int_{\Omega}\left|\nabla\left(u-u_{d}\right)\right|^{2} d x+\frac{\delta}{2} \int_{\Omega} p^{2} d x
$$

subject to

$$
-\Delta u=p \quad \text { on } \Omega .
$$

For the target function $u_{d}\left(x_{1}, x_{2}\right)=\sin \left(\pi x_{1}\right) \sin \left(\pi x_{2}\right)$, we can obtain the exact solution by solving (2.8)-(2.10). The exact solution is

$$
\begin{aligned}
& u\left(x_{1}, x_{2}\right)=\frac{1}{1+2 \delta \pi^{2}} \sin \left(\pi x_{1}\right) \sin \left(\pi x_{2}\right), \\
& p\left(x_{1}, x_{2}\right)=\frac{2 \pi^{2}}{1+2 \delta \pi^{2}} \sin \left(\pi x_{1}\right) \sin \left(\pi x_{2}\right) .
\end{aligned}
$$


The domain is the unit square and we consider a uniform triangulation of $\Omega=(0,1) \times(0,1)$, which consists of the right isosceles.

To investigate the flux approximation property of our algorithm, we consider a subdomain $D=(0,1) \times(0,1 / 2)$ and the approximate flux as follows:

$$
\operatorname{flux}_{D}=\int_{\partial D}\left(\nabla u_{d} \cdot \nu\right) d s \approx \int_{\partial D}\left(\nabla u_{h} \cdot \nu\right) d s .
$$

Note that this approximation is worth for a small $0<\delta<<1$ and it can be a measure of how our approximate solution matches the target function in sense of total flux on a certain region when $\delta$ is small.

As shown in Tables 1-2, the convergence of $u_{h}$ in the energy norm is of order $O(h)$ as predicted in Theorem 4.1 and the convergence of $p_{h}$ is of order $O\left(h^{2}\right)$ for any $\delta>0$, which is better than that predicted in theory, $O\left(h^{2}+\sqrt{\delta} h\right)$. Even though our analysis does not mention on the $L_{2}$ convergence we expect the $O\left(h^{2}\right)$ convergence and it is shown in Tables. Tables 3-4 represents numerical results for nonlinear problem (6.3). We observe desired numerical results. The error in $f_{l u x_{D}}$ seems to be dependent only on $\delta$. Indeed, $C \delta$ is the bound of the error in $f_{l u x_{D}}$ for some constant $C>0$ since the error comes only from the lowest order Crouzeix-Raviart finite element solver for the Lagrange multiplier $\lambda$. Comparing the linear and nonlinear problems we have the same convergence rates for $u$ and $p$. Flux conservation property becomes a little worse because of the existence of a nonlinear term, which can not be approximated in a flux preserving manner.

Figure 1 represents numerical results obtained by changing control parameters for Example 6.1. It shows that the approximate solutions $u_{h}$ and $p_{h}$ converge to the target solutions as the control parameter $\delta$ becomes smaller.

Example 6.2. Let us consider a nonlinear distributed optimal control problem:

$$
\min _{u \in H_{0}^{1}(\Omega), f} \frac{1}{2} \int_{\Omega}\left|\nabla\left(u-u_{d}\right)\right|^{2} d x+\frac{\delta}{2} \int_{\Omega} p^{2} d x
$$

subject to

$$
-\Delta u+10 u^{2}=p \quad \text { on } \Omega .
$$

The target function is $u_{d}\left(x_{1}, x_{2}\right)=\sin \left(\pi x_{1}\right) \sin \left(\pi x_{2}\right)$. Since the exact solution $u$ is not known, we take $u_{h}$ and $p_{h}$ with $h=1 / 96$ as the exact solutions.

Table 3: Numerical results for Example 6.2: $\delta=10^{-4}$

\begin{tabular}{|r||c|c|c|c|c|c|c|}
\hline$n$ & $\left\|u-u_{h}\right\|_{0}$ & $\alpha$ & $\left|u-u_{h}\right|_{1}$ & $\alpha$ & $\left\|p-p_{h}\right\|_{0}$ & $\alpha$ & $\operatorname{err}\left(\right.$ flux $\left._{D}\right)$ \\
\hline 4 & $9.7005 \mathrm{e}-02$ & & $4.4260 \mathrm{e}-01$ & & $2.4881 \mathrm{e}+00$ & & $1.3995 \mathrm{e}-02$ \\
\hline 8 & $2.4979 \mathrm{e}-02$ & 1.96 & $2.1796 \mathrm{e}-01$ & 1.02 & $6.7758 \mathrm{e}-01$ & 1.88 & $1.4760 \mathrm{e}-02$ \\
\hline 16 & $6.2912 \mathrm{e}-03$ & 1.99 & $1.0860 \mathrm{e}-01$ & 1.01 & $1.7441 \mathrm{e}-01$ & 1.96 & $1.4746 \mathrm{e}-02$ \\
\hline 32 & $1.3748 \mathrm{e}-03$ & 2.19 & $5.4260 \mathrm{e}-02$ & 1.00 & $4.5164 \mathrm{e}-02$ & 1.95 & $1.4701 \mathrm{e}-02$ \\
\hline
\end{tabular}


Table 4: Numerical results for Example 6.2: $\delta=10^{-6}$

\begin{tabular}{|r||c|c|c|c|c|c|c|}
\hline$n$ & $\left\|u-u_{h}\right\|_{0}$ & $\alpha$ & $\left|u-u_{h}\right|_{1}$ & $\alpha$ & $\left\|p-p_{h}\right\|_{0}$ & $\alpha$ & $\operatorname{err}\left(\right.$ flux $\left._{D}\right)$ \\
\hline 4 & $9.7965 \mathrm{e}-02$ & & $4.4575 \mathrm{e}-01$ & & $2.4431 \mathrm{e}+00$ & & $1.4129 \mathrm{e}-04$ \\
\hline 8 & $2.5241 \mathrm{e}-02$ & 1.96 & $2.1942 \mathrm{e}-01$ & 1.02 & $6.7969 \mathrm{e}-01$ & 1.85 & $1.4932 \mathrm{e}-04$ \\
\hline 16 & $6.3579 \mathrm{e}-03$ & 1.99 & $1.0921 \mathrm{e}-01$ & 1.01 & $1.7601 \mathrm{e}-01$ & 1.95 & $1.4915 \mathrm{e}-04$ \\
\hline 32 & $1.3898 \mathrm{e}-03$ & 2.19 & $5.4504 \mathrm{e}-02$ & 1.00 & $4.4924 \mathrm{e}-02$ & 1.97 & $1.4802 \mathrm{e}-04$ \\
\hline
\end{tabular}

\section{References}

[1] S. Brenner and L. R. Scott, The Mathematical Theory of Finite Element Methods, Springer-Verlag, Berlin, New York, 1994.

[2] A. Fursikov, Optimal Control of Distributed systems, Theory and Applications, American Mathematical Society, Providence, RI, 2000.

[3] D. Gilbarg and N. S. Trudinger, Elliptic Partial Differential Equations of Second Order, Springer, 1998.

[4] M. Gunzburger, Perspectives in Flow Control and Optimization, SIAM, Philadelphia, 2003.

[5] Max D. Gunzburger and H. C. Lee, Analysis, approximation, and computation of a coupled solid/fluid temperature control problem, Comput. Methods Appl. Mech. Engrg. 118 (1994), no. 1-2, 133-152.

[6] Y. Jeon, E.-J. Park, Nonconforming cell boundary element methods for elliptic problems on triangular mesh, Appl. Numer. Math. 58 (2008), no. 6, 800-814.

[7] Y. Jeon and D. Sheen, Analysis of a cell boundary element method, Adv. Comput. Math. 22 (2005), no. 3, 201-222.

[8] J. L. Lions and E. Magenes, Non-Homogeneous Boundary Value Problems and Applications. Vol. I, Springer, New York, 1972.

[9] A. Quarteroni and A. Valli, Numerical Approximation of Partial Differential Equations, Springer, 1994.

YOUNGMOK JEON

Department of Mathematics

AJou UnIVERSity

SuwON 443-749, Korea

E-mail address: yjeon@ajou.ac.kr

Hyung-Chun LeE

Department of Mathematics

AJou UnIVERSITY

Suwon 443-749, Korea

E-mail address: hclee@ajou.ac.kr 